\title{
A Practical Approach to the Assessment and Management of Psychiatric Emergencies
}

\author{
J. Gregory Kyser, M.D. \\ Vanderbilt University Medical School, Nashville, Tennessee \\ Bradley C. Diner, M.D. \\ Vanderbilt University Medical School, Nashville, Tennessee \\ Gilbert W. Raulston, M.D. \\ Vanderbilt University Medical School, Nashville, Tennessee
}

Follow this and additional works at: https://jdc.jefferson.edu/jeffjpsychiatry

Part of the Psychiatry Commons

Let us know how access to this document benefits you

\section{Recommended Citation}

Kyser, M.D., J. Gregory; Diner, M.D., Bradley C.; and Raulston, M.D., Gilbert W. (1989) "A Practical Approach to the Assessment and Management of Psychiatric Emergencies," Jefferson Journal of Psychiatry. Vol. 7 : Iss. 2 , Article 13.

DOI: https://doi.org/10.29046/JJP.007.2.011

Available at: https://jdc.jefferson.edu/jeffjpsychiatry/vol7/iss2/13

This Article is brought to you for free and open access by the Jefferson Digital Commons. The Jefferson Digital Commons is a service of Thomas Jefferson University's Center for Teaching and Learning (CTL). The Commons is a showcase for Jefferson books and journals, peer-reviewed scholarly publications, unique historical collections from the University archives, and teaching tools. The Jefferson Digital Commons allows researchers and interested readers anywhere in the world to learn about and keep up to date with Jefferson scholarship. This article has been accepted for inclusion in Jefferson Journal of Psychiatry by an authorized administrator of the Jefferson Digital Commons. For more information, please contact: JeffersonDigitalCommons@jefferson.edu. 


\title{
A Practical Approach to the Assessment and Management of Psychiatric Emergencies
}

\author{
J. Gregory Kyser, M.D. \\ Bradley C. Diner, M.D. \\ Gilbert W. Raulston, M.D.
}

\section{INTRODUCTION}

Patients present to the psychiatric emergency room manifesting a wide variety of symptoms ranging from mild anxiety to violent, uncontrollable behavior. The emergency room psychiatrist must recognize these various presentations and provide appropriate interventions for such patients. It may not be of the utmost importance to distinguish between the subtypes of schizophrenia or endogenous vs. exogenous depression, just as it is not crucial to decide immediately the cause of an acute abdomen, primarily to recognize its presence. However, it is critical to recognize the presence of a psychosis, suicidal intent or the presence of an organically caused alteration in mental status. These are, in essence "triage" decisions. In fact, the concordance rate between emergency room and final diagnoses other than alcoholism is no higher than $62 \%$ (1). According to Bassuk (2), "the immediate goal of any emergency evaluation is to identify and manage life-threatening or potentially life-threatening problems, including out-of-control behaviors, serious and chronic self-neglect and severe medical problems either co-existing with or causing psychiatric symptoms." The psychiatrist must have acute management strategies and disposition decisions in mind so that within the initial phases of the interview, impressions regarding the need for restraint and/or psychoactive medication can be put into action. In less emergent situations or after appropriate measures have been taken to ensure safety and control, more time can be devoted toward a complete evaluation.

Many patients will present with less emergent problems for which the basic principles of crisis intervention may apply. They are: setting limited goals, employing focused problem solving, expecting the individuals or family to take some action on their own behalf, providing support for that action and building self reliance and self esteem. In order to facilitate this, one must establish rapport, take charge, assess problems and assets and facilitate closing and follow-up (3).

After gathering the patient's history and providing appropriate counseling, a disposition must be arranged. Referral to a Mental Health Center or a private psychiatrist may be the most desirable plan. Hospitalization is indicated when 
the patient cannot be responsible for his own actions, community resources are unavailable, or there is no social support system.

\section{VIOLENCE, SEVERE AGITATION AND ACUTE PSYCHOSIS}

Violence, severe agitation and acute psychosis often occur together and frequently involve similar methods of management $(2,4,5,6,7)$. The first step is to obtain as much history as possible to assess the degree of potential danger. Anyone accompanying the patient should be interviewed to determine what events led to this presentation and obtain any pertinent past history. Questions regarding past violence, alcohol or other substance abuse, problems with impulse control, the presence of psychosis or cognitive impairment or available methods of violence may alert one to potential danger.

The psychiatrist must then briefly assess the patient's mental status for hypervigilence, hyperactivity or signs of intoxication. If the patient appears to be actively hallucinating, tense, agitated, extremely paranoid or disoriented, caution should be exercised (5). Some experts recommend placing the patient between the door and the interviewer so that the patient does not feel as trapped. Others have opted for a more equidistant approach. The authors recommend that the interviewer locate himself between the patient and the door, some distance from the patient. This allows a quick retreat and will make both feel less trapped. The door should always remain open with psychotic or agitated patients. In the absence of guiding empirical data, the clinical situation and one's own intuition are probably the best indicators.

If verbal measures fail, physical restraint may be necessary. Optimally, one is assisted by five individuals - one for each limb and one (usually a nurse) free to administer medication, close doors, etc. The psychiatrist should never take part in any patient restraint, but rather give orders and direct the action. In most instances, restraint alone will be sufficient intervention. However, to ensure patient safety and facilitate management, medication may be needed. In these cases, there are generally only two alternatives; antipsychotic or anxiolytic agents. If the goal is simple sedation, an antipsychotic may not be needed; benzodiazepines may well fulfill this purpose while preserving the underlying psychopathology for further evaluation. The authors recommend lorazepam for the following reasons: In doses of $1-4 \mathrm{mg}$, it is short acting, rapidly and predictably absorbed intramuscularly (unlike other benzodiazepines), and does not depend upon hepatic metabolism.

In other patients, lorazepam may not be suitable and/or sufficient. The use of antipsychotics and the technique of rapid neuroleptization may be more appropriate (8). Rapid neuroleptization refers to the parenteral administration of antipsychotics given at 30-60 minute intervals. The high-potency agents, in spite of their higher incidence of extrapyramidal side effects (E.P.S.), are preferable due to their lower tendency to produce significant orthostatic hypotension and anticholinergic side effects. Haloperidol is the prototypic high-potency 
anti-psychotic used for rapid neuroleptization; one to four injections can be given in doses of $2.5-10 \mathrm{mg}$.

Recently this technique has been questioned $(9,10)$. Positron emission tomography data (11) suggest that haloperidol doses as low as $8 \mathrm{mg}$ per day saturate 90 percent of $\mathrm{D}_{2}$ dopamine receptors. High dose neuroleptics may serve only to increase sedation and the risk of toxicity without shortening hospitalization time. The authors prefer to limit total doses of haloperidol to $10-20 \mathrm{mg}$ in the emergency setting. If sedation is the goal, $50 \mathrm{mg}$ of diphenhydramine may be given IM along with the neuroleptic; this has the added benefit of prophylaxis against E.P.S. If further sedation is required, intramuscular lorazepam may be then substituted for haloperidol and diphenhydramine.

Caution should be employed in patients with suspected phencyclidine (PCP) intoxication or anticholinergic psychosis. Benzodiazepines are the medication of choice for PCP intoxication. Neuroleptics should be used prudently because they may exacerbate muscle spasm and the intrinsic anticholinergic activity of PCP. Physostigmine can be used with close medical monitoring in anticholinergic delirium.

Many violent or psychotic patients will meet legal criteria for commitment. These criteria vary somewhat among states, but if a patient is a potential danger to himself or others, or if he is so gravely disabled that he could not make rational, safe decisions, commitment is probably indicated. This is especially true if the patient is unwilling to admit himself voluntarily. In such cases, it is often preferable to medicate a patient as little as possible initially, in order to allow a second (referred) psychiatrist to make a more complete evaluation of the demonstrated psychopathology. All too often, committed patients arrive at their final destination so heavily medicated that an evaluation must be made on history alone; thus, the importance of documenting the initial mental status cannot be overemphasized.

Not every psychotic patient need be admitted to a hospital. Many can be managed as outpatients, particularly if they have a good social support system. Outpatient follow-up should be arranged as soon as possible. If an appointment is scheduled in 1-2 days, anti-psychotic medications may not be needed. In cases which are delayed or where treatment is indicated more urgently, the emergency room physician can prescribe a low dose anti-psychotic medication. Haloperidol, in doses of 5-10 mg twice daily, is usually adequate. One must remember the potential to develop extra-pyramidal symptoms and consider prescribing an anti-parkinsonian medication, such as benztropine 1-2 mg bid or trihexyphenidyl $2 \mathrm{mg}$ bid.

\section{VIOLENCE AND SUBSTANCE ABUSE}

Primary psychiatric illnesses are not the only causes of acute agitation or psychosis. Stimulants such as amphetamines may mimic a paranoid psychosis. Cocaine may cause a variety of psychiatric symptoms including euphoria, dyspho- 
ria, psychosis and delirium (12). The delirium is characterized by excitation and the acute onset of disturbed attention and perception, impaired cognition, disorientation, visual hallucinations and illusions $(12,13)$. Other signs include hyperthermia, mydriasis reactive to light, tachycardia and occasionally seizures (13). The presence of fresh needle marks in addition to these signs should alert the physician to the possibility of cocaine-induced psychosis. A positive urine drug screen confirms the diagnosis. Cocaine intoxication is a medical emergency which may result in sudden death (13). When it is suspected, medical personnel should be alerted immediately. Consideration should then be given to the use of IV diazepam or lorazepam to control the agitation and the administration of IV propranolol to antagonize the sympathomimetic effects of cocaine (14).

Other substance-induced causes of acute psychosis include PCP psychosis (15), neuroleptic malignant syndrome and organic brain syndrome secondary to alcohol intoxication or withdrawal.

\section{STRESS AND ANXIETY}

Stress and anxiety are common complaints of patients presenting to an emergency room. Associated symptoms range from generalized anxiety, insomnia and dysphoria, to suicidal ideation. One must decide whether the symptoms are due to a primary psychiatric illness or whether they represent a reaction to stress in a previously well-functioning individual (16). Adjustment to a situational crisis can be extremely debilitating; in severe cases, the patient may experience cognitive disorganization or suicidal ideation. In mild cases, patients may present with nervousness or anxiety, indecisiveness, and sadness. They come in because they are "at the end of their rope" and cannot seem to structure their life, resolve simple problems or interact with others appropriately. The history may or may not identify significant life stressors. Since patients usually come in at the point of optimal stress, they are generally very amenable to crisis intervention.

Medication may be helpful to provide temporary, symptomatic relief of insomnia or disabling anxiety until formal follow-up is arranged. A short-acting anxiolytic or sedative is usually sufficient to carry a patient until an outpatient appointment may be scheduled. In mild cases, hydroxyzine can provide temporary sedation, has little abuse potential, and has few side effects. $50-100 \mathrm{mg}$ IM or PO may be given in the emergency room, followed by $50 \mathrm{mg}$ PO tid. In other cases, hydroxyzine will not be sufficient. In these cases, benzodiazepines are the best pharmacologic intervention. Lorazepam (1-3 mg per day) or alprazolam (.5 to $1 \mathrm{mg}$ per day) are the most popular anti-anxiety agents used. Geriatric patients require lower doses, generally. In these low doses, there are few side effects, lethal overdose is almost impossible, and they provide excellent acute relief of anxiety or insomnia. Some prefer triazolam or flurazepam for sleep but caution should be exercised using flurazepam in a geriatric patient, due to its longer half life. 


\section{DEPRESSION/SUICIDE}

Evaluation and management of suicidality is one of the most common situations encountered in the emergency room. While many patients may fit the pattern of a high- or low-risk patient, decisions are ultimately based on clinical intuition. However, it is more reliable if based on a solid, educated assessment of risk of genuineness of suicidal intent.

Suicide cuts across all diagnoses, but the following have been shown to be associated with a higher percentage of completed suicides: depression (30\%), alcoholism (15\%), schizophrenia (10\%)—particularly those with a recent diagnosis and under 30 years of age; opiate addiction (10\%), personality disorder (5\%) (17). Among psychiatric patients, risk factors include middle age (30-40's) male sex, white race, undesirable life events, especially humiliating ones or loss of a significant person. Suicide tends to occur early in the clinical course of psychiatric patients and thus at a younger age than the general population. In contrast, suicide among alcoholics tends to be a late sequela (18). Hospitalized patients are at greatest risk during the inpatient period and for 6-12 months after discharge (18). Any previous suicide attempt places a patient at a greater risk of suicide. In addition, any medical illness (regardless of psychiatric diagnosis) feelings of helplessness and hopelessness, the presence of a detailed plan, recent loss (actual or perceived), suicidal fantasies, increasing social isolation, and an inability to accept help increases the suicide risk particularly if the patient has been treated within the previous 6 months (19).

In the general population, individuals at greatest risk are white, male; over age 45; separated, widowed or divorced; living alone; and unemployed. While men complete suicide more often, younger women attempt it three times more frequently (20). The suicide rate for those 75 and older remains approximately double the rate for those aged 45-54. There has been a rapid increase in the suicide rate for young people over the last 20 years. Although the rate has risen in this group it still occupies a small fraction of the total number of cases, contributing little to the overall rate.

The following mnemonic may be helpful to assess suicidal risk. The originators suggest that hospitalization be strongly considered if five to six of the ten items are positive (21).

Sex (male)

Age (under 20, over 45)

Depression (or other major psychiatric illness)

Previous attempt

Ethanol abuse

Rational thinking loss

Social supports lacking

Organized plan 
No spouse

Sickness

The two most common methods are self-poisoning or self-inflicted wrist lacerations. Pill ingesters usually have less serious psychiatric illness, the actions are more impulsive and there is usually more than one type of pill taken (22).

Regarding patients with borderline personality disorder Perlmutter (23) notes that, "Such patients are well known to utilize a disproportionate amount of crisis services and to be extraordinarily difficult to manage at times of crisis." They often present with anxiety, suicidal ideation or an actual suicide attempt. Life stresses for borderlines are usually related to actual or perceived abandonment, and the suicidal act (often wrist-cutting) is designed to prevent a separation from an ambivalently held love object (23). Self-harm is often the patient's way of relieving the tension and intolerable affects of the experience. Indeed, many borderline patients will feel better after such an act. The ability of these patients to elicit anger, guilt and dread in the emergency room staff is well recognized. Borderline patients frequently make physicians not only the target for their internal rage, but lead them to experience that rage firsthand. When evaluating these patients, one must be mindful of countertransference issues and its influence on disposition planning. Essentially, the same rules for admitting any suicidal patient hold. When in doubt, the safest decision is to admit. However, repeatedly hospitalizing borderline patients may reward their regressive, self-destructive behavior and thus increase the risk of future suicide gestures and attempts. Referral back to their support is preferable if at all possible. One should encourage borderline patients to seek help through psychiatric emergency services when they feel out of control, but it should be made explicit that emergency room visits will usually not end in admission and that hospitalization may not be beneficial (23).

Regardless of how depressed or anxious a patient is, there are several drugs which should rarely be dispensed in the emergency room. These include tricyclic antidepressants, monoamine oxidase inhibitors and barbiturates. Some clinicians believe that there are selected cases where tricyclic antidepressant medication is appropriate in the emergency room $(8,24)$. The authors feel that if the patient is sick enough to require any of these agents immediately, hospitalization is probably indicated.

\section{GERIATRIC EMERGENCIES}

By the year 2000, an estimated 33 million people or $25 \%$ of the population will be over 65 . As many as $10-20 \%$ of the elderly possess a "significant degree of memory defect, disorientation, decline in intellectual performance, or frank psychiatric disorder" (25). Elderly patients usually present when the patient is the sickest, the support system is most strained, and the problem is most complicated (25). For many, a psychiatric referral represents their first contact 
with mental health services. For others, the referral may be the first step toward eventual nursing-home placement or other institutional care (26). For these reasons, accurate diagnosis, effective management, and appropriate disposition are critical. Unfortunately, these goals often prove elusive due to a lack of awareness among clinicians of the multifaceted relationships between medical and psychiatric illnesses in the elderly, and a misunderstanding of the psychosocial aspects of aging (26). Compared with younger patients, the elderly often have no previous psychiatric illness and are more likely to be referred by a third party (26).

Waxman, et al, reported that as many as $40 \%$ of geriatric psychiatric emergency room evaluations result in a diagnosis of organic brain syndrome (OBS) with no specific etiology found (26). In fact, a large number of these patients failed to receive the medical evaluation necessary to detect potential organic causes of their psychiatric symptoms (27). Therefore, medical evaluation is probably the single most important intervention that can be provided in the emergency room. The physical exam should be complete, seeking diagnostic evidence such as abnormal vital signs, asterixis, pupillary abnormalities, localizing neurological findings, etc. Particular attention should be paid to medications, medical problems and psychosocial situations. The recent onset of acute confusional states with visual hallucinations, disorientation and/or poor selective attention should alert the psychiatrist to the possibility of delirium. A differential diagnosis including malignancy, metabolic disorders, infections, and drug effects must be considered (28). The Folstein mini-mental status exam (29) is an excellent screening tool and is easily and briefly administered. As with most emergency psychiatric patients, a drug screen can be beneficial.

Paranoid disorders and depression are also common in elderly patients. While depression in the elderly may have many of the same symptoms as in younger groups, there are some important differences (27). Concentration, memory disturbance, and disorientation can be associated symptoms which prevent patients from seeking help. Vague persecutory or delusional thinking may also be a sign of depression in the elderly, but it could also suggest an early dementia. These complaints should not be taken lightly and, while emergency treatment may not be necessary, appropriate follow-up is vital. Herst (28) has provided several case studies which highlight some of the more common emergency room presentations that one might encounter with a geropsychiatric patient.

\section{EMERGENCY CHILD PSYCHIATRY}

Children present special problems for the psychiatric emergency room. The beginning psychiatrist may have anxieties related to the interview itself due to the presence of demanding parents and the lack of an optimal disposition. Children and adolescents present unique emergency psychiatric problems, and they often arouse feelings of inadequacy in those new to their care. While adults 
usually present because of their own suffering, children usually present because of their parents' discomfort. Understandably, children often minimize their difficulties and, initially, resist interventions. Adults usually present for failures in work or love or for intolerable symptoms, while children usually present because of unacceptable behavior, such as running away, violence, suicide attempts, fire-setting, sexual activity, substance abuse or academic problems (30).

Someone must take the child to the emergency room, and that someone is usually a parent, most often a parent in crisis. Crisis intervention techniques, while similar to those for adults, must include specific considerations for children (31). The child or adolescent often oscillates between overemphasis and denial of the reported difficulty. One often sees prominent scapegoating, both parent to child and child to parent. It is important to consider the parents' embarrassment in bringing their child to the psychiatric emergency room and the resultant narcissistic injury. With younger children it is often easier to interview the parent first; older children and adolescents are best interviewed before the parents. Similarly, the younger the child, the more one needs to rely on the family for factual data. One may gain valuable information by viewing the family together and by spending at least a few minutes with the family as a whole.

The child interview should be divided into three segments (32). One first directs the child to the interviewing room noting how the family separates (or resists separation) as well as the initial display of trust or mistrust between child and therapist. As with adults, the first portion of the child interview should be open-ended. The interview then shifts to discussing how the child is doing currently, then moves to play and fantasy issues. Children are usually easily engaged in drawing, and the themes often relate to the child's presenting difficulty. The interviewer may draw half a picture and the child is asked to complete the picture, tell a story about the picture and give the picture a title. Other techniques include asking the child to draw his or her family, to name three wishes, and to name their biggest worry. Displacement may be helpful (32) for example, when the child states, "I don't worry," the therapist may reply, "Oh, well I see that you don't worry. I wonder what you would worry about if you did worry." The final portion of the interview should be more directive and should include a question regarding the child's reason for coming to the emergency room. This can be omitted if it is clear that the child does not know (32). As in adult emergency psychiatry, rapid assessment and disposition are more imperative than perfect diagnostic clarity. The decision whether or not to admit depends on whether or not the support system can deal well enough with the situation.

Considerable caution should be exercised in medicating children. Consent from the parents must be obtained before prescribing any medication and the lowest effective dose should be used. As with adults, one would rarely begin a tricyclic antidepressant in the emergency room. The same would apply to methylphenidate and benzodiazepines as well. 


\section{ALCOHOL ABUSE}

The emergency room prevalence of alcoholism is $20 \%$; the nighttime prevalence is $29 \%$ and the daytime prevalence is $11 \%(33)$. The psychiatric disorders most frequently associated with alcoholism are depression and antisocial personality disorder. Interestingly, alcohol-related problems are the chief complaints in only $8 \%$ of alcoholics in emergency rooms.

The four CAGE (34) questions are easily remembered and provide a useful screening device for assessing the presence and severity of alcohol abuse. They include: 1) Has the patient made attempts to Cut down? 2) Has he been Annoyed by criticism of his drinking? 3) Does he feel Guilty about drinking? 4) Does he resort to Eye openers to suppress withdrawal symptoms or hangovers? Also important are questions to detect the presence of an alcohol withdrawal syndrome (AWS) or help to predict the probability that one will occur. Inquiries as to what the patient drinks, how much, how often and for what lengths of time help to define severity. Other questions which help to predict an AWS include whether a patient has ever experienced seizures, delirium tremens, hallucinations, or blackouts. A history of significant alcohol withdrawal symptoms in the past will often predict a subsequent episode. One should also distinguish between daily vs. episodic "binge" drinking and ask about the longest period of sobriety over the past 12 months.

It is customary to distinguish an "early or minor withdrawal" from a "late or major withdrawal," i.e., delirium tremens (35). "Minor" withdrawal can be quite variable depending on the intensity and duration of alcohol intake. The earliest symptoms are insomnia, vivid dreaming and "hangover." Anxiety, mild agitation, anorexia, tremor, sleeplessness, mild tachycardia (less than 100), and hypertension (greater than 150/90) appear a few hours after cessation of drinking and disappear within 48 hours. This syndrome occurs after persistent ethanol consumption of approximately one pint of liquor or twelve beers per day and may not need pharmacological intervention. In "major" withdrawal, the previous symptoms are gradually followed by increasing psychomotor agitation, autonomic hyperactivity, disorientation, confusion, and auditory or visual hallucinations. Disorientation and confusion are the essential hallmarks of delirium tremens. Seizures frequently precede such a reaction, although they may occur in patients who do not progress to major withdrawal. Major withdrawal can be lethal and is an indication for hospitalization and detoxification.

The routine administration of thiamine $100 \mathrm{mg}$ IM will help prevent the occurrence of the Wernicke-Korsakoff Syndrome. Patients in early withdrawal can frequently be managed as outpatients. It is not unreasonable to provide several tablets of a long-acting benzodiazepine such as diazepam (10-20 mg) or chlorodiazepoxide (50-100 mg) to carry them over to an appointment. Anyone who is intoxicated can usually manage for several hours without fear of significant withdrawal and can safely be released without medication. If a patient is kept in the emergency room to "sober up," aspiration precautions and frequent 
checks should be instituted. Any recognized alcohol abuser should be referred to an appropriate rehabilitation program.

\section{CONCLUSION}

Patient encounters should begin with triage decisions, assigning priority to more emergent problems such as delirium and agitation. In order to provide optimal benefit the emergency room psychiatrist must have the breadth of knowledge and flexibility to recognize and treat acute emergencies while still being adept with crisis-oriented psychotherapeutic modalities. Disposition decisions will vary according to local availability but still call for informed, rational thought. The need for a diversity of skills ranging from medicine to case work makes the psychiatric emergency room an ideal setting for the biopsychosocial approach.

\section{REFERENCES}

1. Liebermann PB, Baker FM: The reliability of psychiatric diagnosis in the emergency room. Hosp Community Psychiatry (3):291-293, 1985

2. Bassuk EL: The diagnosis and treatment of psychiatric emergencies. Comp Ther 11(7):6-12, 1985

3. Puryear DA: Crisis intervention. Manual of Psychiatric Consultation and Emergency Care Guggenheim, FG, Weiner, MF Eds. 33-42 Jason Aronson, Inc. 1984

4. Rada RT: The violent patient: Rapid assessment and management. Psychosomatics 22(2):101-109, 1981

5. Lenvenson LJ: Dealing with the violent patient. Postgrad Med 78(5):329-335, 1985

6. Tupin JP: The violent patient: A strategy for management and diagnosis. Hosp Community Psychiatry 34(1):37-40, 1983

7. Jacobs D: Evaluation and management of the violent patient in emergency settings. Psychiatric Clin of North Am 6(2):259-269, 1983

8. Jacobs D: Psychopharmacologic management of the psychiatric emergency patient. Gen Hosp Psychiatry 6:203-210, 1984

9. Coffman J, et al: Clinical effectiveness of oral and parenteral rapid neuroleptization. J Clin Psychiatry, 48(1), 1987

10. Tupin J: Focal neuroleptization: An approach to optimal dosing for initial and continuing therapy. J Clin Psychopharmacol 5(3 Sup.):155-215, 1985

11. Farde L, Hall H, Ehrin E, Seduall G: Quantitative analysis of $D_{2}$ domamine receptor binding in the living human brain by PET. Science 231(4735):258-61, 1986

12. Van Dyke C, Byck R: Cocaine: 1884-1974, in Cocaine and Other Stimulants, Ellinwood EH and Kilby MM, Eds. New York, Plenum Press, 1976

13. Wetli CV, Fishbain DA: Cocaine-induced psychosis and sudden death in recreational cocaine users. J Forensic Sci 30(3):873-880, 1985

14. Rappolt RT, Gay GR, Inaba DS: Propranolol: A specific antagonist to cocaine. Clin Toxicol 109(3):265-267, 1977

15. McCarron MM, et al: Acute phencyclidine intoxication: Clinical patterns complications and treatment. Ann Emerg Med 10(6):290-297, 1981 
16. Rabin PL, Hussain G: Crisis intervention in an emergency setting. Ann Emerg Med 12(5):300-302, 1983

17. Miles CP: Conditions predisposing to suicide: A review. J Nerv Ment Dis 164:231246, 1977

18. Hirschfield RM, Davidson L: Clinical risk factors for suicide. Psychiatric Ann 18(11):628-635, 1988

19. Hillard JR, Ramm D, Zung WK, Holland JM: Suicide in a psychiatric emergency room population. Am J Psychiatry 140(4):459-462, 1983

20. Jacobs D: Evaluation and care of suicidal behavior in emergency settings. Int J Psychiatric Med 12(4):295-310, 1982-83

21. Patterson W, et al: Evaluation of suicidal patients: The SAD PERSONS scale. Psychosomatics 24(4), 1983

22. Robins LN, Kulbok PA: Epidemiological studies in suicide. Psychiatric Ann 18(11): 619-626, 1988

23. Perlmutter RA: The borderline patient in the emergency department: An approach to evaluation and management. Psychiatric Quart 54(3):190-196, 1982

24. Brodsky L, Pieczynski B: The use of antidepressants in the psychiatric emergency department. J Clin Pharmacol 5(1):35-38, 1985

25. Bassuk EL, Minden S, Apsler R: Geriatric emergencies: psychiatric or medical. Am J Psychiatry 140(5):539-542, 1983

26. Waxman HM, Carner EA, Dubin W, Klein M: Geriatric psychiatry in the emergency department: Characteristics of geriatric and non-geriatric admissions. J Am Geriatr So $30(7): 427-432,1982$

27. Waxman HM, Dubin W, Klein M, Weiss KJ, Carner EA: Geriatric psychiatry in the emergency department, II: Evaluation and treatment of geriatric and non-geriatric admissions. J Am Geriat So 32(5):343-349, 1984

28. Hearst LD: Emergency psychiatry for the elderly. Psychiatr Clin of North Am 6(2):271-280, 1983

29. Folstein M, Folstein S, McHugh P: Mini-Mental State: A practical method for grading the cognitive state of patients for the clinician. J Psychiatr Res (12): 189-198, 1975

30. Turgay A: Psychiatric emergencies in children. Psychiatr J Univ Ottawa 7(4):254259,1982

31. Garfinkel BD: Suicide attempts in children and adolescents. Am J Psychiatry 139(10): 1257-1261, 1982

32. Werkman SL: The psychiatric diagnostic interview with children. Am J of Orthopsychiatry 35:764-771, 1965

33. Rund DA, Summers WK, Levin M: Alcohol use and psychiatric illness in emergency patients. JAMA 245(12):1240-41, 1981

34. Ewing J, Mayfield D: CAGE. Am J Psychiatry 131:1121-1123, 1974

35. Naranjo C, Sellers E: Clinical assessment and pharmacotherapy of the alcohol withdrawal syndrome. Advances in New Psychopharmacological Treatments for Alcoholism 265-281, Elseiver Science Pub., 1985 